\title{
Anorexia nervosa, paternalism and clinical practice
}

\author{
Gustavo Costa Medeiros ${ }^{1}$, Daniela Guimarães Sampaio ${ }^{1}$, Felipe Corchs ${ }^{1}$ \\ 1 University of São Paulo Medical School (FMUSP), Department and Institute of Psychiatry, São Paulo, SP, Brazil.
}

Received: 7/10/2014 - Accepted: 7/23/2014

DOl: 10.1590/0101-60830000000028

\section{Dear Editor}

Anorexia nervosa (AN) is an eating disorder whose therapeutic interventions usually produce unsatisfactory results. This disorder is characterized by high rates of relapse, tendency to chronicity, high morbidity and mortality ${ }^{1}$. Low motivation is a central issue that hinders $\mathrm{AN}$ treatment ${ }^{1}$. Interpersonal relationships seem to be crucial in AN and may play a role in this sense as well. We propose that specific patterns of interpersonal interactions can arise when professionals are dealing with AN subjects and that this peculiar rapport is not only a characteristic of the disorder in question, but could also be a part of what perpetuates and aggravates the problem.

Those who interact with AN patients can refer feelings of helplessness, fear, anxiety and hopelessness ${ }^{2}$, pointing to a possible degree of aversivity in these relationships. This fact may be aggravated by the slow improvement typical of AN, which can be frustrating and, as a result of this, professionals can adopt a strong and directive attitude toward the patients trying to reach better outcomes in the treatment. In fact, a coercive attitude in AN can generate relatively quick results ${ }^{3}$, what can provide a transient attenuation of professional's discomfort. This may have reinforcing effects over the controlling attitude, making it more likely to occur in future similar occasions. In other words, too much directivity appears to relief professional's distress and, therefore, negatively reinforce coercive posture, but it may also have adverse consequences to the patient's treatment ${ }^{4}$.

Fallouts are noticed as a remarkably paternalistic approach seen, for example, in procedures such as involuntary hospitalization not based on scientific guideless or arbitrary tube feeding - tends to increase the already high resistance in these patients ${ }^{5}$. An intimidating posture also can punish potentially unpleasant statements made by the patient, possibly suppressing genuine reports and predisposing to false descriptions of improvement. Coercion-generated improvements may be volatile and fragile and even unfavorable in the long term. For instance, forced hospitalizations decrease the voluntary search of treatment ${ }^{3}$, which is a major issue since the motivation to change is an important element for therapeutic success ${ }^{1}$.
A healthy rapport seems to have an important role in reaching a favorable outcome in treatments since a mutual and reliable alliance in $\mathrm{AN}$ increases the chance of a continuous and more effective approach ${ }^{4}$. However, according to Darcy et al. ${ }^{6}$ the therapist-patient alliance is yet relatively under-researched especially when studied from the professional perspective and there is a deficiency of mental health professional's continued training on the use of rational paternalistic techniques ${ }^{3}$.

In short, some evidence suggests that a directive posture is more common in AN compared to other mental disorders and may have unfavorable effects over its treatment. It seems to be important to establish a positive therapeutic alliance to optimize motivation in this disorder. Prioritize cooperation in AN apparently can shifts the focus from control to an efficient therapeutic bond. Further research on the professional-patient relationship in AN cases is needed in other to elucidated this point and enhance the success of interventions.

\section{References}

1. Espíndola CR, Blay SL. Long term remission of anorexia nervosa: factors involved in the outcome of female patients. PLoS One. 2013;8(2):e56275.

2. Gabbard GO. Chapter 12 - Substances related disorders and eating disorders. In: Gabbard GO. Psychodynamic psychiatry in clinical practice. $4^{\text {th }}$ ed. Washington, DC: American Psychiatric Publishing; 2006. p. 259-82.

3. Seo MK, Kim SH, Kyu RM. Coercion in psychiatric care: can paternalism justify coercion? Int J Soc Psychiatry. 2013;59(3):217-23.

4. Horvath AO, Del Re AC, Flückiger C, Symonds D. Alliance in individual psychotherapy. Psychotherapy (Chic). 2011;48(1):9-16.

5. Bischoff MM, Terence JG. The client resistance predicted by therapist behavior: a study of sequential dependence. J Couns Psychol. 1995;42(4):487-95.

6. Darcy AM, Katz S, Fitzpatrick KK, Forsberg S, Utzinger L, Lock J. All better? How former anorexia nervosa patients define recovery and engaged in treatment. Eur Eat Disord Rev. 2010;18(4):260-70. 